\title{
Reflets
}

Revue ontaroise d'intervention sociale et communautaire

\section{Des noms qui en disent long...}

\section{Nérée St-Amand}

Volume 6, numéro 1, printemps 2000

Approches d’intervention : définir et renouveler nos pratiques

URI : https://id.erudit.org/iderudit/026294ar

DOI : https://doi.org/10.7202/026294ar

Aller au sommaire du numéro

Éditeur(s)

Reflets : Revue ontaroise d'intervention sociale et communautaire

ISSN

1203-4576 (imprimé)

1712-8498 (numérique)

Découvrir la revue

Citer cet article

St-Amand, N. (2000). Des noms qui en disent long.... Reflets, 6(1), 36-63.

https://doi.org/10.7202/026294ar

Tous droits réservés (C) Reflets : Revue ontaroise d'intervention sociale et communautaire, 2000

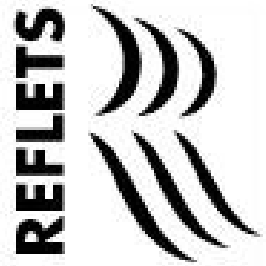

Ce document est protégé par la loi sur le droit d'auteur. L'utilisation des services d'Érudit (y compris la reproduction) est assujettie à sa politique d'utilisation que vous pouvez consulter en ligne.

https://apropos.erudit.org/fr/usagers/politique-dutilisation/ 


\title{
Des noms qui en disent long...
}

\author{
Nérée St-Amand, \\ professeur, École de service social, Université d'Ottawa
}

Dire, c'est plus que préfigurer l'action ou la rationaliser, c'est déjà agir, opter pour une stratégie, et c'est surtout exclure (Michel Autès 1992).

\section{Introduction}

Au cours de mes quelques trente années de pratique professionnelle et d'enseignement,j'ai souvent été frappé par les mots que les professionnels et les professionnelles de l'intervention utilisent pour désigner la "clientèle» des services sociaux et de la santé. Des termes tels cas, patients, malades et clients, pour ne mentionner que ceux-là, se retrouvent très régulièrement dans les textes de formation en service social et sur les lèvres des praticiennes et des praticiens, comme d'ailleurs dans l'ensemble de la littérature des professions vouées à l'aide professionnelle. Combien de fois n'avons-nous pas entendu ou utilisé des expressions telles : "C'est un cas intéressant», "Ce client est difficile», "Cette patiente me draine beaucoup»? Combien de fois n'avonsnous pas apposé un vocable diffamatoire à une famille en difficulté, comme par exemple "cette famille est désengagée, négligente, résistante, difficilement approchable»? Quels critères utilisons-nous pour nommer des personnes ou qualifier des familles qui vivent dans la misère? Il me semble que, dans la littérature en service social et plus particulièrement dans le champ de la relation d'aide, on s'est peu penché sur la question de l'étiquetage des gens. 
Par ailleurs, au cours des années 1970, nous avons été témoins de l'amorce d'une remise en question, plus ou moins radicale, de la façon de nommer les gens, tendance initiée principalement par les mouvements de contre-culture dont le mouvement féministe et celui des personnes ayant connu la psychiatrie institutionnelle. En lien avec les pratiques de conscientisation et de désinstitutionnalisation qui prévalaient alors, un ensemble d'autres termes font leur apparition tels consommateurs, survivants, sujets et victimes, etc. Ces termes remettent en question, de façon plus ou moins voilée, plus ou moins radicale, la façon de nommer les gens et les bases inégalitaires de l'intervention professionnelle. En écho à ces nouvelles façons de définir les rapports entre professionnels et clientèle, de nombreux mouvements visant à redonner du pouvoir et à habiliter les personnes (empowerment) ont mis en évidence le potentiel des gens plutôt que leurs handicaps ou leurs limites. Ainsi, de nouvelles façons de nommer émergent, qui tentent d'établir un autre rapport entre aidants et «aidables». Ce dernier terme n'assume plus que la personne est «aidée», comme la littérature le suggérait jusque-là. Il sème le doute sur une relation qui présumait en quelque sorte qu'une personne était aidante et l'autre aidée (Platteau 1988).

Aujourd'hui, nous faisons face à des malaises et même à des tensions importantes vis-à-vis les façons de désigner la "clientèle» des professions d'aide. Certaines écoles continuent de la désigner en se référant à un modèle d'inspiration médicale (cas, patients, clients, malades), alors que d'autres, inspirées par les analyses féministes ou structurelles en particulier, rompent avec ces façons de nommer et insistent sur la notion d'acteurs (de Gaulejac et Roy 1993) plutôt que sur celle de sujets passifs. Plusieurs vocables s'affrontent donc, utilisant divers critères, plus ou moins définis, pour désigner les gens auprès de qui interviennent des professionnelles et des professionnels de l'aide. C'est à la fois aux noms utilisés et, également, aux critères et aux points de repères qui génèrent ces façons de nommer que cet article s'intéresse.

En partant de certaines études classiques de l'étiquetage social, ce texte propose de passer en revue un certain nombre de termes puisés de la littérature de la relation d'aide, de mes recherches et 
de ma pratique. L'exemple des familles à problèmes multiples servira d'illustration principale à mes remarques qui seront par la suite étendues à deux autres "clientèles", soit les femmes et les personnes psychiatrisées. Je tenterai de démontrer que les noms utilisés reflètent la distance entre les uns et les autres et, par conséquent, identifient la déviance des uns et la supériorité des autres. Par la suite, d'autres vocables seront présentés, axés principalement sur les préoccupations de clientèle. Je conclurai cet article en proposant certaines polarités qui peuvent servir à analyser les façons de nommer les gens.

\section{Nommer et marginaliser...}

Dans son étude classique The Outsider, Howard Becker définit la déviance de la façon suivante.

Social groups create deviance by making rules whose infraction constitutes deviance and by applying those rules to particular people and labelling them as outsiders. From this point of view, deviance is not a quality of the act the person commits, but rather a consequence of the application by others of rules and sanctions to an "offender". The deviant is one to whom the label has successfully been applied; deviant behavior is behavior that people label (Becker, cité in Wright 1984 : 62).

Cette définition illustre bien que la déviance soit une construction, un produit et non un acte en soi. Becker souligne ici une des formes de contrôle exercées par ceux et celles qui ont le pouvoir d'évaluer certains comportements, certaines attitudes et de les qualifier comme étant plus ou moins acceptables. La déviance est ici nommée, désignée, et c'est cette étape du processus qui nous intéresse particulièrement. À son tour, Gove insiste sur l'interaction entre la personne qui a le pouvoir de désigner et celle qui se voit apposer une étiquette. 
To labelling theorists, deviance is not a quality of an act, but instead is produced in the interaction between a person who commits an act and those who respond to it. In summary, the labelling theorists have focused on the societal attributes of those who react and those who are reacted against in order to explain why certain persons and not others are labelled as deviant. They argue that once a person has been labelled a deviant - and particularly if that person has passed through a degradation ceremony and been forced to become a member of a deviant group - the person experienced a profound and frequently irreversible socialization process. He or she has not only acquired an inferior status, but has also developed a deviant worldview and the knowledge and skills that go with it. And perhaps equally important, he or she has developed a deviant self-image based upon the image of himself or herself received through the actions of others (Gove: 10).

«...en désignant une personne comme "cliente», nous imposons en quelque sorte une distance thérapeutique et un rapport de domination entre le désignant et le désigné..."
Par exemple, en désignant une personne comme «cliente», nous imposons en quelque sorte une distance thérapeutique et un rapport de domination entre le désignant et le désigné, entre la personne qui possède certaines connaissances et quelques formes d'expertise, habituellement académiques et professionnelles, et celle qui vit une expérience, que nous nommerons pour l'instant, patiente, car, comme nous le confiait une personne ayant connu la psychiatrie, «il faut être bien patient pour survivre dans un tel système». De telles étiquettes ne tiennent pas compte des contextes de pauvreté, d'oppression, de violence dont sont victimes les personnes ainsi désignées. Ils s'attardent uniquement à décrire un acte (le délinquant, le pédophile, l'agressif, l'hyperactif) ou un état, "an attribute that is deeply discrediting", pour reprendre l'expression de Goffman (1975).

Une fois étiquetée, l'entourage immédiat de ces personnes se fait complice plus ou moins conscient de l'expertise professionnelle et exerce diverses formes de pression sur les individus ainsi marqués afin qu'ils et qu'elles maintiennent cette image. Lorsque le titre de malade mental est apposé à quelqu'un, plusieurs études 
"...une personne est souvent récompensée pour avoir adopté le rôle stéréotypé qui accompagne le diagnostic.» démontrent que la tendance à agir en fonction de cette étiquette augmente.

Labeling theory states that the cultural stereotype of mental illness acts as a self-fulfilling prophecy — others react toward the potential patient in a uniform way that leads him to play the expected role, and thus tends to validate the original definition. The mentally ill role may become the only way the individual can cope with the situation (Jones et Cochrane 1981 : 100).

Qui plus est, une personne est souvent récompensée pour avoir adopté le rôle stéréotypé qui accompagne le diagnostic. C'est le cas des gens qui collaborent avec les formes de traitements recommandés ou imposés par la psychiatrie, mais c'est aussi le cas des indemnités financières accordées sous forme d'assistance ou de pension suite aux diagnostics d'incompétence (p. ex.incapacités de travail). Une personne ayant surmonté ses problèmes psychiatriques et ayant réussi un sevrage complet de psychotropes me confiait qu'elle ne peut plus se qualifier pour l'assistance financière qu'elle recevait lorsqu'elle prenait servilement ses médicaments. Une autre, accidentée de travail, faisait de peine et de misère, un peu de bicyclette pour renforcer son dos. Dénoncée, elle a été exclue du programme d'indemnisation. Il semble que les experts et les institutions réagissent plutôt mal aux changements de rôle et au manque de soumission de la part de celles et de ceux qu'ils soignent...

Le fait d'être pris et stigmatisé comme déviant a des conséquences importantes sur la participation ultérieure à la vie sociale et sur l'évolution de l'image de soi de l'individu. La conséquence principale est un changement dans l'identité de l'individu aux yeux des autres; (il acquiert un nouveau statut. On a découvert une personnalité différente de celle qu'on lui prêtait. Il sera donc étiqueté comme pédé, drogué, maniaque ou cinglé et traité en conséquence (Becker 1963 : 54-55). 
"Une fois étiquetée, la participation des personnes à la vie économique et sociale devient fort limitée.»
Une fois étiquetée, la participation des personnes à la vie économique et sociale devient fort limitée. Un schizophrène sera reconnu tel, à condition qu'il adopte le plan de traitement et la médication qui vient avec la désignation. Dans le comportement des "familles pathologiques», des «inaptes», des «incapables de s'organiser», on sélectionnera les incidents qui décrivent et prouvent leur diagnostic. L'étiquetage constitue ainsi un rapport de domination et implique la non-reconnaissance des individus concernés comme des citoyens et des citoyennes à part entière. Le diagnostic peut donc s'analyser comme un rapport de force qui crée et maintient des formes plus ou moins voilées de domination, par le biais de l'expertise.

Les personnes qui font l'objet de cette recherche [...] ont également en commun le fait d'appartenir à ces couches sociales composées de gens dont on parle beaucoup — «ces gens-là» — mais que l'on entend peu. Et c'est ici que les systèmes de marquage fondés sur les diagnostics de la pathologie psychosociale jouent un rôle fondamental dans la mesure où ils constituent le dispositif symbolique qui consacre la première mise à l'écart. L'individu ainsi marqué devient un cas, un risque, une statistique. La logique du processus diagnostique aliène, dépossède l'individu de son savoir sur lui-même, au profit d'un savoir professionnel et technique (Blais 1998 : 29).

L'individu qui est victime de cette expertise n'est plus une personne, mais un cas; il n'est plus un acteur, mais un patient identifié, marqué; il passe d'un statut de citoyen à celui de client, de malade. Et s'il parvient, à la force du poignet, à se sortir de ce carcan, on continuera de lui rappeler que son état antérieur le poursuit comme une ombre, en le désignant comme un «expatient», un «ex-psychiatrisé», un «ex-prisonnier», un «former mental health patient" ou encore comme une "ex-prostituée», etc. L'individu est ainsi confiné à vie, en quelque sorte, à son étiquette. 
"...la personne

étiquetée comme déviante adopte, souvent par la force des choses, un comportement conforme aux attentes sociales. En ce sens, l'étiquetage change notre perception de l'individu et modifie le concept de soi de cette personne.»
Nous pouvons parler de stigmatisation au sens actif et de l'effet dévastateur de la désignation sous une étiquette infériorisante et infamante, qui dépossède l'individu des droits les plus élémentaires (Diederich 1991 : 13).

Selon Wolfensberger et Tullman (cité dans Diederich 1991), les réactions de l'entourage de la personne étiquetée déterminent son comportement. Il s'agit d'une prophétie auto-réalisatrice : la personne étiquetée comme déviante adopte, souvent par la force des choses, un comportement conforme aux attentes sociales. En ce sens, l'étiquetage change notre perception de l'individu et modifie le concept de soi de cette personne. On n'a qu'à penser aux personnes ayant eu un épisode psychiatrique et qui veulent se présenter pour obtenir un emploi. Une des questions qui leur est inévitablement posée à la personne, consiste à lui demander d'expliquer les trous dans son cheminement de carrière, l'obligeant ainsi à renouer avec cet épisode difficile de sa vie. Il en va de même pour les gens ayant séjourné en prison ou ayant fait une cure de désintoxication. De Gaulejac et Roy (1993) suggèrent que ces comportements font écho, en nousmêmes, à notre propre crainte de nous retrouver ainsi : désigner et nommer ce dont on a peur, ce qu'on veut éviter pour soi et son entourage...

Dans la suite de ce texte, nous présenterons quelques illustrations de gens qui, lorsqu'ils s'écartent de la norme, sont la cible de plusieurs formes d'étiquetage. Pour démontrer les nombreux vocables en usage, nous ferons un survol de la littérature et de l'histoire du travail social auprès des familles dites à problèmes multiples et parlerons rapidement de deux autres groupes ciblés : les femmes, plus particulièrement les mères et les personnes ayant vécu une expérience en psychiatrie. 


\section{Les familles chaotiques...}

- À quoi servent les noms qu'ils portent, demanda le Moucheron, s'ils n'y répondent pas?

- Ils ne servent à rien pour eux, répondit Alice, mais je suppose qu'ils sont utiles aux gens qui les nomment (Lewis Carroll, cité dans Kirk et Kutchins 1998 : 11).

Dès son origine, le service social est confronté à deux façons radicalement opposées de concevoir les familles pauvres. Pour Jane Addams, les familles défavorisées se retrouvent dans la classe ouvrière et vivent dans la pauvreté à cause des effets négatifs de l'économie marchande, de la déstabilisation suite aux conflits armés (la Guerre de 1914-1918 vient de finir) et du manque de services sociaux adéquats. Inspirée par des analyses socialistes, elle propose, dès les années 1920, des réformes démocratiques et des politiques d'urbanisme qui tiennent compte des structures de marché et de l'analyse du pouvoir qui défavorisent et oppriment les plus pauvres. En fait, son analyse démontre que seules de grandes réformes socio-politiques et économiques peuvent résoudre les problèmes sociaux des familles défavorisées. À l'opposé, Mary Richmond, à la même époque, perçoit les familles «instables ou dégénérées» comme faisant preuve d'un manque de solidarité et de cohésion familiale, résultat de leurs inaptitudes ou de leurs incompétences. Inspirée par l'analyse médicale qui prévaut alors, elle centre sa pratique du service social sur les individus et les familles vivant des situations socio-économiques difficiles. À l'encontre de Addams, elle écrit beaucoup, proposant dans ses études de cas, des mesures qui visent à changer cette "clientèle» plutôt que de remettre en question le déséquilibre des forces socio-politiques et économiques.

La suite de l'histoire des professions d'aide doit beaucoup à Sigmund Freud et à la perspective psychanalytique qui vient littéralement camper les personnes souffrantes et défavorisées dans un rapport de clientèle. On délaisse les analyses de Jane Addams pour se concentrer sur l'intra-psychique, en inscrivant les 
«La littérature sur les approches systémiques comporte de nombreuses allusions à un étiquetage très dévalorisant et condescendant face aux familles vivant habituellement dans des contextes de pauvreté extrême.» problèmes de pauvreté et de marginalisation dans une optique médicale de diagnostic individuel. Le courant dominant de la psychanalyse, en service social comme dans les autres professions d'aide, contribue au développement de la culpabilité, à la construction de modèles psychologico-centriques de l'intervention (Corin et al. 1990: 255), menant même à une remise en question du rôle des femmes en service social. La désignation des familles défavorisées illustre ces tendances.

Les familles chaotiques, les familles à anorexiques mentaux, les parents de psychotiques, les parents de boulimiques, les familles à enfants obèses, les familles difficilement approchables, voici quelques-uns des termes recensés pour nommer les familles qui font face à de multiples problèmes. Les années 1950, entre autres, ont été témoins d'une série d'études et de projets visant à étudier la situation des familles dites «à problèmes multiples» et à répondre à leurs besoins. Overton (1953), Birt (1956), Tinker (1959), Ayers et Lagey (1970) et Geismar et Wood (1960) emploient plusieurs expressions pour les désigner: familles résistantes, familles problèmes, désorganisées, difficiles à rejoindre, milieux familiaux pathogènes. Une dysfonction au sein de l'unité familiale et une résistance à l'aide caractérisent ces désignations. En se référant à des situations de négligence ou d'abus, Noller et Fitzpatrick (1993) utilisent le terme de parents délaissants, Clément (1993) celui de familles impossibles, fétichisées et de parents maltraitants. À leur tour, Belpaire (1994) les qualifie de familles enchevêtrées, Caille (1985) de familles dysfonctionnelles, de familles chaotiques et de familles anorexiques et Minuchin (1974) de familles psychosomatiques.
Le schéma de la famille psychosomatique de Minuchin se retrouve chez les familles à anorexiques mentaux avec les mêmes enchevêtrements de relations, de sur protection, de rigidité, d'incapacité à régler les conflits détaillés à propos des familles à enfant obèse ou boulimique (Apfeldorfer 1993 : 172).

La littérature sur les approches systémiques comporte de nombreuses allusions à un étiquetage très dévalorisant et 
condescendant face aux familles vivant habituellement dans des contextes de pauvreté extrême. Une dysfonction au sein de la famille (p. ex. le milieu familial pathogène de Caille (1985)) et une résistance à l'aide offerte par les agences (on retrouve régulièrement les expressions familles désengagées ou familles résistantes) ressortent de la littérature des familles dites à problèmes multiples.

Si une symbiose entre les orientations de Richmond et le travail avec les familles à problèmes multiples est largement présente dans la littérature et constitue le courant dominant dans le domaine de la relation d'aide, il n'en va pas de même pour l'approche de Addams. Avant les années 1975, très peu d'auteurs réussiront à lier la notion des familles à problèmes multiples aux conditions socio-politique et économique de la pauvreté. C'est à partir de cette époque que nous assistons à une période de rupture et de contestation sociale du courant dominant, portée plus particulièrement par les mouvements féministe et antipsychiatrique. Ceux-ci remettent en question les structures du pouvoir et certaines des assises de l'intervention professionnelle. La désignation des personnes évolue rapidement à cette époque, grâce aux nombreux écrits qui rejettent plus ou moins radicalement les approches professionnelles ou qui visent, à tout le moins, un éveil de conscience et une prise en main chez les personnes défavorisées. Ainsi, les psychiatrisés se retrouvent en dehors des murs de l'institution. Ils deviennent visibles à plusieurs niveaux, signent des écrits, produisent des films et des vidéos, revendiquent leurs droits. Leur littérature témoigne de l'oppression vécue sous l'égide

"...la notion d'acteur social prend plus de place en service social, ce qui remet en question le rôle passif accordé aux personnes victimes des rapports thérapeutiques opprimants.» de la psychiatrie institutionnelle. Par ailleurs, les femmes accèdent plus nombreuses que jamais au marché du travail rémunéré et revendiquent des politiques égalitaires en matière d'emploi. Elles exposent et dénoncent les problèmes de la violence dite conjugale, de même que les traitements habituellement imposés aux personnes victimes. Grâce à l'analyse de Autès (1992), la notion d'acteur social prend plus de place en service social, ce qui remet en question le rôle passif accordé aux personnes victimes des rapports thérapeutiques opprimants. Ainsi,il est possible de repérer dans les noms utilisés pour étiqueter les femmes et les personnes 
ayant un vécu psychiatrique, plusieurs des courants à peine esquissés ici.

\section{Les mères étouffantes}

Les noms attribués aux femmes, et plus particulièrement à celles qui ne rencontrent pas les attentes sociales liées à leur rôle de mères, sont souvent diffamatoires dans la littérature des professions d'aide.

Les mères abusives ne sont pas seulement les mères étouffantes ou dévoreuses ou perpétuellement anxieuses: ce sont aussi celles qui ne donnent pas suffisamment de sécurité affective à l'enfant ni ne fournissent de réponses apaisantes à ses demandes inquiètes; les mères rejetantes sont justement celles visà-vis de qui on insiste et auprès de qui on continue à quémander ou à vouloir prouver qu'on est l'enfant bon et acceptable requis. (Caille 1993 : 79)

Les femmes, piliers de la morale judéo-chrétienne et responsables de l'harmonie familiale, ont, comme mères, une marge de manœuvre fort limitée. Les attentes exigées d'elles sont à la mesure des noms qu'on appose à celles qui transgressent les normes. Eiguer fait allusion aux mères menteuses, aux mères illégitimes et aux mères «fantasmantes» (1987 : 43).Van Wormer (1989) utilise le terme de femmes troublées. Clément (1993) parle de mères étouffantes, rejetantes, dévoreuses. Elles sont souvent désignées selon le métier, la place qu'elles occupent ou le motif qui les différencie de la majorité: prostituée, assistée sociale, criminelle, mère illégitime. La littérature présente aussi les femmes comme mensongères, manipulatrices, recherchant l'attention (Worrall 1990).

Nous voilà en train de revivre dans la relation thérapentique avec la famille notre propre illusion, ces moments illusoires des premières relations avec la mère, 
privilège de la prime enfance, où s'instaure ce mouvement de continuité entre la mère et le nourrisson, et que l'on peut traduire par la notion, nous semble-t-il, la plus forte, celle que nous appelons le lien à la mère menteuse, à la mère fantasmante (Eiguer $1987: 43$ ).

"Dans les façons de les diagnostiquer, les femmes vivant avec un conjoint ayant un problème sont souvent blâmées pour la situation de ce dernier.»
Dans les façons de les diagnostiquer, les femmes vivant avec un conjoint ayant un problème sont souvent blâmées pour la situation de ce dernier. C'est ainsi que l'épouse d'un alcoolique sera qualifiée de co-dépendante, en raison du fait que ces femmes «dépendantes» nouent des relations avec des hommes toxicomanes. En conséquence, les caractéristiques de la co-dépendance reflètent souvent le rôle culturellement prescrit pour les femmes. Elles deviennent ainsi partiellement ou totalement responsables des problèmes de consommation du conjoint. À ce vocable, Van Wormer (1989) oppose celui de «survivor's syndrome», désignant ainsi la seule voie mise à la disposition des femmes pour réagir aux problèmes du conjoint dépendant.

L'alimentation et le poids des femmes sont aussi un autre domaine où l'étiquetage est très présent. La littérature portant sur les femmes obèses, anorexiques ou boulimiques, est beaucoup plus nombreuse que celles des hommes dont le poids n'est pas dans les normes ou qui éprouvent des difficultés au niveau de l'alimentation (Fuller et Groce 1991). Les conditions nerveuses des femmes ont également été très exploitées dans les études de cas, comme dans l'expression: elle est sur ses nerfs, anxieuse, hystérique.

\section{Les PVP (personnes ayant un vécu psychiatrique)}

Au cours des ans, les personnes ayant vécu un épisode psychiatrique, en institution ou en dehors des murs, ont été affublées également de vocables diffamatoires. Certes, on leur a appliqué les termes classiques de patients, clients, cas, empruntés à la médecine, mais la liste ne s'arrête pas là. En plus, grâce au Diagnostic 
«...il faut noter qu'un séjour en institution hospitalière et l'évaluation automatique qu'on $y$ mène quant au degré de dangerosité des "psychiatrisés», surtout s'il est jugé élevé, font que les noms les qualifiant revêtent alors un caractère plus permanent.» and Statistical Manual of Mental Disorders (DSM), on les a désignés selon leur diagnostic (schizophrène, psychotique, psychopathe), selon la place qu'ils et elles occupent en institution (le 377, soit le numéro du lit ou de la chambre) et enfin, selon le problème particulier auxquelles ces personnes font face (suicidaire, maniacodépressive, endeuillée chronique, etc.). On utilise aussi couramment d'autres qualificatifs tels troublé, mental, fou, retardé, dérangé, détraqué (St-Amand 1985 : 109-167). Notons également que le verbe utilisé ici est lourd de sens: «il est mental, elle est dérangée, il est chaviré»; ces verbes d'état dénotent peu d'espoir de revirement de la situation problématique. C'est ce qui fera dire à Szasz que la maladie mentale est simplement "une déviation des normes morales, légales et psychosociales» (1976:122). S'inspirant de cette analyse, Rosen propose une reprise de pouvoir des gens concernés, en questionnant les pratiques professionnelles :

The label of mentally ill and the current practice of mental health care has resulted in stigmatization, incarceration, and loss of identity to such an extent that mental health professional practice is tantamount to systematic colonization and disempowerment of the mentally ill. Users of mental health care services must reclaim mental health practice by challenging professional descriptions, practices, and presumptuous ownership of their lives (1994: 132).

Comme dans tout le réseau de santé, les mots des années 1980 introduisent un rapport de consommation entre la clientèle et l'institution psychiatrique. Par exemple, plusieurs individus et organisations utilisent le mot consommateur, associé ou prestataire pour parler de gens suivis par des psychiatres, alors que d'autres les identifient comme des PVP, jeu de mot autour de l'acronyme VIP (Very Important Person). Malgré cet effort visant à donner de l'importance aux personnes, il faut noter qu'un séjour en institution hospitalière et l'évaluation automatique qu'on y mène quant au degré de dangerosité des "psychiatrisés», surtout s'il est jugé élevé, font que les noms les qualifiant revêtent alors un caractère plus permanent. Ce psychotique, cette maniaque, cette 
schizophrène, voilà des termes désignant habituellement l'incurabilité de la personne.

\section{Quelques pistes...}

«En analysant les

divers vocables utilisés

pour désigner les

transgresseurs de

normes, nous

constatons rapidement

que la majorité des

façons de les nommer

sont à la fois

réductionnistes,

durables, diffamatoires

et désengageantes."

"Grâce aux dossiers

professionnels, ce

marquage devient

permanent.»
Que se dégage-t-il de cette brève incursion dans la littérature des étiquettes utilisées pour nommer, classer et marginaliser? En analysant les divers vocables utilisés pour désigner les transgresseurs de normes, nous constatons rapidement que la majorité des façons de les nommer sont à la fois réductionnistes, durables, diffamatoires et désengageantes.

Elles sont réductionnistes dans le sens qu'elles réduisent la personne à son diagnostic, officiel ou pas. On ne parle plus de Marie ou de Pierre comme personne qui a une histoire, un vécu, des valeurs, un héritage culturel, une contribution politique, mais simplement d'une étiquette qui, en principe, englobe l'ensemble de ce qu'elle vit. «Tu parles d'Émerie Leblanc, ils saviont pas qui tu voulais dire; si tu dis Émerie Fou, là, ils saviont!» (St-Amand 1985 :109). Négligées sont les autres dimensions de sa vie, le contexte de pauvreté dont la personne a été victime, la violence qu'elle a subie, les défis et les obstacles qu'elle a surmontés. «Les gens, ce ne sont pas des problèmes, ce sont des gens", nous confiait une personne pauvre travaillant dans un milieu communautaire. Et une autre de renchérir : «Si on fait du bénévolat, y nous croient pas. Si on leur dit qu'on est malade, y nous croient pas. Si on leur dit qu'on est fou, là y nous croient».

Elles sont durables, parce que ces façons de nommer, d'étiqueter, perdurent bien au-delà des moments de crise ou de consultation. Grâce à un arsenal de moyens utilisés tant par les institutions, les professionnels que la communauté, on s'assure qu'un criminel d'un moment le demeure en permanence, qu'une ex-prostituée pourra difficilement effacer son passé ou qu'une famille désengagée garde longtemps son étiquette. Grâce aux dossiers professionnels, ce marquage devient permanent. «Ça fait 
que lui halait une paye de fou", commentait un interviewé, insinuant par là que sa pension était pour ainsi dire permanente et garantie. Pourtant, en désignant les gens ordinaires, on ne dit pas un ex-marié, une ex-opérée à cœur ouvert, un ex-coureur, une ex-endeuillée pour désigner les gens qui vivent des expériences particulières.

Les façons de nommer sont diffamatoires parce que presque tous les termes utilisés dénigrent les personnes et les familles, leur rappellent qu'elles ne sont que des cas, qu'elles ne sont qu'un tas de problèmes, une charge pour leur milieu et les services sociaux. Les familles sont mal adaptées, à risques ou impossibles (Caille, 1985) et dans ces façons de les pointer du doigt, rien de bien positif ne se dégage : aucune reconnaissance de leurs luttes, de leurs expériences, de leurs acquis, de leur ingéniosité.

Ces façons de nommer sont enfin désengageantes au sens où les vocables employés laissent croire que les personnes ne participent aucunement à la désignation et pas davantage au traitement qui s'ensuit. Elles ne sont que des spectatrices qui voient leur sort se jouer dans une arène auxquelles elles n'ont pas accès. Elles sont absentes des conférences de cas qui les concernent. Le diagnostic n'est pas négocié, mais imposé. Il ne tient pas compte de leur potentiel et de leur créativité. Il stigmatise, et pour longtemps, les victimes d'un incident, de circonstances, de pauvreté, de précarité. En ce sens, il perpétue la pauvreté et la précarité.

Je suis pénalisée, démunie, impuissante, sans liberté de choix, sans droit à l'autonomie, surveillée, dévalorisée, toujours abaissée, humiliée. [...] La pauvreté n'est pas uniquement un manque d'argent. La pauvreté c'est le manque de dignité, le manque de respect, le manque de choix. La logique des gestionnaires est de nous abaisser un peu plus quand nous avons un peu d'espoir de nous en sortir. Pas moyen de remonter la pente (StAmand et Kérisit 1998 : 67).

Ces quatre facteurs combinés font en sorte que la personne est dépossédée de son pouvoir et qu'il lui est extrêmement difficile, 
«L'étiquette est ainsi transmise comme une forme d'héritage social, de statut permanent.» de même que sa famille, de se voir autrement, d'agir autrement. L'étiquette est ainsi transmise comme une forme d'héritage social, de statut permanent. «Le monde l'appelle le fou, c'est comme ça qu'est son nom. C'est de même qu'ils l'ont baptisé par icitte", nous confiait une personne au sujet de quelqu'un qui avait séjourné en institution psychiatrique.

\section{Pistes pour un renouvellement des pratiques}

«Passer du rôle de client à celui de créateur et de producteur, d'objet ou de cas à celui de sujet connaissant et participant aux diverses étapes de sa reprise de pouvoir, voilà certains principes qu'il nous semble important de respecter.»
La grande question alors est de savoir comment parvenir à un meilleur accès des individus aux institutions, comment provoquer une plus grande ouverture de cellesci tout en sauvegardant une certaine clarté des rôles et une visibilité du pouvoir. L'un des moyens, sans doute, est d'accentuer le travail de conscientisation afin de redonner aux personnes concernées la force de réagir collectivement comme sujets de droit plutôt que comme objets de prise en charge (Ghysens $1986: 39$ ).

Comment peut-on faire autrement? Que faire pour se départir de pratiques diffamatoires, discriminatoires, opprimantes? Est-il nécessaire, comme le suggère la citation, de sauvegarder une certaine visibilité du pouvoir? Quels mots peut-on utiliser pour désigner les gens qui souffrent de problèmes, auxquels on a apposé un diagnostic et qui tentent de s'en sortir pour le mieux, dans des contextes de pauvreté et d'oppression fort contraignants? Cette citation de Ghysens suggère quelques pistes que nous considérions importantes : actions collectives et conscientisation étant des pratiques que nous estimons essentielles à des changements véritables, et non pas seulement à un changement cosmétique d'appellations conformes à la rectitude politique (politically correct). Passer du rôle de client à celui de créateur et de producteur, d'objet ou de cas à celui de sujet connaissant et participant aux diverses étapes de sa reprise de pouvoir, voilà certains principes qu'il nous semble important de respecter. 
Inspirée par Jane Addams, Meyer (1973) s'est élevée contre les pratiques discriminatoires en affirmant que les familles marginalisées ont en commun une grande pauvreté et un isolement social. Elle propose des réformes sociales qui doivent s'adresser aux inégalités socio-économiques grandissantes. Pour elle, le traitement des problèmes psychosociaux doit passer par une compréhension du vécu socioculturel de ces familles.

De nombreuses ressources communautaires tentent de valoriser le travail des familles pauvres (St-Amand et Kérisit 1998). Le vocabulaire utilisé par ces groupes est très différent, voire opposé à celui recensé dans la littérature professionnelle. On parle ici de survivants, de victimes, de participants, d'actrices, de personnes, de groupes de solidarité. On décrie les mots qui tuent, la stigmatisation, les pratiques discriminatoires, les termes diffamatoires. Le volume Dis-moi qui tu aides propose des termes qui peuvent inspirer nos pratiques tels : aidés, membres, personnes, intéressés, citoyens, coopérants et partenaires pour désigner, selon les circonstances, les gens ayant connu une période difficile. "Cette aide, faite de relation, d'amitié et de respect est finalement un échange où chacun donne et reçoit, différemment mais également» (Ghysens 1986 : 29).

\section{Quelques repères pour établir une typologie de l'aide}

Il $y$ avait une femme qui faisait de l'orientation professionnelle avec des gens qui participaient à un programme financé par le gouvernement fédéral; elle travaillait avec des personnes aux prises avec divers problèmes de santé mentale; elle travaillait avec un grand nombre de femmes qui faisaient tout simplement une dépression. "Tout cela c'est de la foutaise, a-t-elle dit. Ces femmes-là ne devraient pas rentrer dans la définition médicale de la psychiatrie; ce sont tout simplement des femmes qui en arrachent dans la vie». 
Et puis elle a ajouté : "Ce que ça prend, ce sont des services qui nous permettent de faire une analyse féministe des problèmes et qui nous aident à travailler avec les femmes sans les classer dans un modèle médical». Elle a donc préparé une proposition et c'est ainsi que notre centre est né... (St-Amand et Kérisit $1998: 33)$

Que peut-on dégager de cette nomenclature utilisée pour désigner la clientèle qui a recours à l'aide professionnelle? Les quelques remarques qui suivent cherchent à dégager quelques constats de l'étude préliminaire entreprise jusqu'ici.

Il existe peu de repères : chaque champ de pratique et chaque profession semble évoluer sans que nous ne possédions de repères généralement acceptés pour tenter de nommer les gens désignés comme la clientèle des services professionnels.

L'analyse du savoir-pouvoir est souvent absente des discours professionnels. On assume que d'aucuns ont le savoir et que les autres ont besoin de cette expertise, de soins, d'aide. Des noms comme aidants, professionnels d'un côté, et patients, malades de l'autre, établissent déjà les rapports de domination, mais sans le proclamer. Les pratiques d'autonomie, de résistance sont ainsi évacuées, ignorées.

La neutralité professionnelle et la distance entre la personne aidante et l'individu qu' elle veut aider semblent souvent des facteurs importants, bien que peu explicites. Il existe une plus

«Des termes tels "patient», "parent désengagé» ou «cas» ont tendance à éloigner bien plus qu'à favoriser le rapprochement et proposer des liens de collaboration, sinon de thérapie.» grande distance entre l'aidant et l'aidé que celle qui existerait entre des membres, des participants ou des partenaires. Des termes tels "patient», "parent désengagé» ou "cas» ont tendance à éloigner bien plus qu'à favoriser le rapprochement et proposer des liens de collaboration, sinon de thérapie.

Une des questions qui se pose est de savoir s'il est possible de réconcilier l'irréconciliable. Existe-t-il des points de médiation entre les approches structurelles et les approches psychosociales? Entre la vision de Jane Addams et celle de Mary Richmond? Entre l'acteur et le cas? Peut-on tenir compte à la fois des connaissances des uns sans dénigrer les savoirs et les pratiques 
des autres? Les expertises professionnelles sont-elles nécessairement bâties sur l'oppression structurelle des pauvres et des laissés pour compte, des marginalisés? Peuvent-elles s'ouvrir à des dimensions de médiation, de collaboration, de partage, d'échange ou de conscientisation, comme celles que nous observons dans les mouvements d'entraide ou ceux des cuisines collectives? Les quelques remarques qui suivent pourraient peutêtre alimenter nos réflexions et inspirer nos pratiques visant à nommer sans marginaliser. Elles proposent différentes manières de voir les gens qui consultent les professionnels de l'aide.

Au départ, les noms que nous avons inventoriés semblent se rattacher à deux pôles principaux : le pôle objet et le pôle sujet (Tableau 1), représentés respectivement par Richmond et Addams au départ. Nous sommes conscients que la présentation qui suit, tend plutôt à opposer ces deux courants. Il n'en reste pas moins qu'il existe entre eux de nombreuses zones d'affinité et que les façons de voir comme les interventions, peuvent comporter un certain mixage qu'un schéma parvient difficilement à rendre.Ainsi, ce schéma se veut plutôt une illustration des tensions et des polarités qui nous permettra de dégager quatre perspectives, quatre modèles qui modulent la relation d'aide. 
Tableau 1

Polarités de l'aide

\section{Le pôle objet}

- L'expertise, la science

- Les savoirs académiques ou professionnels

- Le patient - la malade

\section{Le pôle sujet}

- La vulgarisation

- Les savoirs d'expérience

- L'acteur - l'actrice

\section{Conséquences de ces deux façons de voir}

- La domination

- L'assujettissement

- La neutralité

- Des diagnostics diffamatoires

- De la dépendance à l'indépendance
- La prise en charge

- La reconnaissance

- L'engagement

- Auto-diagnostic, conscientisation des conditions d'oppression

- L'interdépendance

\section{Sources d'inspiration}

- Le modèle médical

- Les associations et corporations

- Les approches psychosociales

- Les approches systémiques familiales
- Les approches de conscientisation

- Les regroupements alternatifs

- Les approches féministes, anti-psychiatriques et radicales

- Les approches structurelles, de solidarité et d'entraide

\section{Quelques exemples de noms}

- Patient, client, consommateur, cas

- Familles à problèmes multiples, enchevêtrées
- Survivante, acteur, victime, personne, créateur, producteur, pair-aidants

- Familles-ressources, familles engagées

De ce tableau, suggérant certaines polarités, certaines tensions entre diverses façons de voir les gens, nous tenterons de dégager quatre modèles de l'aide, selon l'analyse que révèlent les noms utilisés pour désigner les personnes. Ces quatre façons de voir sont un éventail des tendances selon certains repères idéologiques. 


\section{A. Modèle paternaliste}

«La tendance à utiliser des termes condescendants... relève d'une approche paternaliste de voir les gens.»
La tendance à utiliser des termes condescendants, tels patients, clients, cas, handicapés, femme troublée, malade, etc., relève d'une approche paternaliste de voir les gens. Ces termes reflètent un regard distant, hautain, et une relation asymétrique, généralement à sens unique entre aidants et aidés.

\begin{abstract}
La littérature anthropologique recourt fréquemment à l'expression de "patronage» ou de "relations patronclient" pour désigner des rapports asymétriques ou des hiérarchies informelles entre individus ou groupes d'individus dans les sociétés traditionnelles. On peut dégager quelques grandes caractéristiques des relations «patron-client». D'abord, il s'agit de relations fondées sur la réciprocité et l'inégalité: entre le patron et le client qui sont liés par des droits et devoirs réciproques, il $y$ a inégalité de statut, de richesse et de pouvoir (Plattean 1986: 189-190).
\end{abstract}

\section{B. Modèle de partenariat}

«Ce modèle de partenariat suggère que les professionnels tiennent compte, dans une certaine mesure, de la réalité des personnes qui les consultent.»
Plusieurs autres noms désignent les gens en invitant une certaine collaboration entre aidants et aidés et un rapport de force moins inégal que le précédent. Il se reflète dans des vocables tels: membre, personne, partenaires, aidables. Ce modèle de partenariat suggère que les professionnels tiennent compte, dans une certaine mesure, de la réalité des personnes qui les consultent. «Aidez-nous à vous aider», suppliait un psychiatre qui avait peur que les psychiatrisés rejettent totalement le modèle psychiatrique.

Au-delà d'une aide technique, les personnes attendent du travailleur social que celui-ci les rejoigne dans ce qu'elles vivent comme difficultés et luttes quotidiennes. 
Derrière la première demande se cache très souvent un besoin de reconnaissance, de valorisation. [...] Il s'agit alors d'un type d'aide très différent de l'aide matérielle ou technique dans laquelle l'échange était fondamentalement inégal. Dans cette nouvelle forme d'accompagnement ou "guidance", l'échange entre les partenaires est beaucoup plus important, même si, ici encore le statut professionnel de l'aidant définit clairement les possibilités et les limites du rôle (Ghysens 1986:30-31).

Plusieurs associations professionnelles et de nombreux milieux de pratique professionnelle, tout en s'inscrivant généralement dans ce modèle, jouent sur plusieurs cordes, prétendant vouloir «remettre» le pouvoir aux gens, mais ne leur accordant qu'une participation symbolique aux tables de concertation ou aux prises de décision. Un comité de douze ou quinze personnes, où siège une ou deux personnes représentant la "clientèle» en est un exemple.

\section{Modèle de conscientisation}

"Ce modèle de conscientisation implique une analyse socio-politique des situations vécues, des conditions d'oppression et laisse une plus grande marge de manouvre aux gens.»
Ce troisième modèle dénote une prise de conscience du rapport de domination entre aidants et aidés et reflète dans les termes utilisés une analyse socio-politique de la situation. Les mots utilisés ici dénotent une prise de conscience et une reprise du pouvoir de la part des personnes et des familles qualifiées d'aidées. Les termes tels victime, survivante, opprimée, établissent une distance entre le diagnostic et la personne. Ils dénotent une remise en question des rapports professionnels et suggèrent d'autres façons de voir et de faire, à l'extérieur du rapport thérapeutique. Ce modèle de conscientisation implique une analyse socio-politique des situations vécues, des conditions d'oppression et laisse une plus grande marge de manœuvre aux gens. 
Le système carcéral produit un objet social, gérable, utilisable : la délinquance. [...] La délinquance, c'est un système de significations, un objet social produit par les pratiques sociales pénitentiaires, et dont la fonction est de gérer socialement les écarts normatifs. Le délinquant est un produit d'institutions. L'échec de la prison est en fait sa réussite. La même analyse peut s'appliquer au travail social. [...] Le travail social contribue ainsi à produire un objet social, qu'on peut appeler la marginalité et qui est la socialisation, l'effet des pratiques de travail social sur l'ensemble des comportements d'écart à la norme sociale qu'on peut ressembler sous le terme de pauvreté. Là où la prison opère sur le délit, sur la faute, le travail social opère sur l'écart, la différence (Autès 1992 : 39-40).

Si la majorité des groupes d'entraide nourrissent des affinités certaines avec le modèle de conscientisation, une minorité adopte une position plus neutre, en développant des pratiques quasi professionnelles et en s'identifiant davantage au modèle précédent. Cela dit, les cuisines collectives et les groupes d'alphabétisation utilisent généralement un modèle de conscientisation lors de l'analyse des liens hiérarchiques (de classe, de sexe, de culture, entre autres) entre aidants et aidés.

\section{Modèle radical}

"Le modèle radical démystifie et rejette les rapports

thérapeutiques...»
Le quatrième modèle est à caractère plus radical en ce sens qu'il remet en cause les fondements mêmes de la relation professionnelle. Le modèle radical démystifie et rejette les rapports thérapeutiques, en utilisant une grille structurelle. Il dénonce les rapports inégaux, les fondements illusoires du savoir objectif et questionne la raison d'être des interventions professionnelles. Inspirés par plusieurs réseaux antipsychiatriques, de nombreux auteurs et auteures ont démontré que la psychiatrie n'a rien à 
voir avec la santé mentale (Szasz 1976; Cooper 1970; Breggin 1991), que la médecine n'a rien en commun avec la santé (Illich 1975; Chesler 1979), que les savoirs professionnels et académiques masquent tout simplement des relations de pouvoir. Ces façons de voir affirment que l'oppression, la violence, la pauvreté et la marginalisation expliquent bien davantage les malaises des gens que le DSM ou les autres façons de diagnostiquer ou de classer. Une analyse institutionnelle, féministe radicale ou structurelle, est habituellement à la base de ces façons de concevoir les rapports sociaux. C'est ici que nous retrouvons des termes tels citoyens, acteurs, victimes, personnes. Le rejet de toutes formes de vocables discriminatoires est important pour comprendre les débats structurels inhérents à cette façon de voir.

Malgré l'appel à la notion de "citoyen-décideur» contenue dans les politiques et discours actuels, la «nouvelle santé publique» reste, sur le plan de la recherche, imprégnée du modèle épidémiologique et pathogénique ou déficitaire pour désigner les problèmes et les besoins. En enfermant la subjectivité des gens dans un ordre objectif et objectivant, ce modèle contribue à occulter les enjeux collectifs sous-jacents à la problématique de la santé mentale et participe ainsi au processus d'une double exclusion : celle des lieux de la parole où sont définis les besoins; celle des actions par lesquelles les citoyens deviennent acteurs dans les processus décisionnels les concernant (Blais 1998 :30).

En guise de conclusion :l'aide est-elle un mensonge? Un mythe? Une nécessité?

Ce n'est sans doute pas un hasard si, dans deux langues occidentales (le grec et l'allemand anciens), les mots «don» et «poison» ont la même racine. Lorsqu'on parle d'aide, on commet donc un mensonge social; on veut créer l'impression du désintéressement là où, en réalité, des intérêts sont en jeu. Le recours à l'image de l'aide fait donc partie des formes utilisées pour créer l'illusion 
de la générosité et du désintéressement là où l'acte est en fait intéressé, et celle de l'égalité là où la relation est en réalité profondément asymétrique, c'est-à-dire finalement pour dissimuler la relation de pouvoir qui s'établit au travers d'échanges d'aide non équivalents (Platteau 1986: 193-194).

Dans ce texte, nous avons tenté de démontrer que les termes utilisés pour désigner les personnes en quête d'aide ne sont pas neutres, mais qu'au contraire, ils sont porteurs de sens. Client ne rime pas avec acteur; l'utilisation de termes tels patient et opprimé ne dénotent pas la même conception des gens. Nous estimons qu'une grille d'analyse de ces façons de nommer est nécessaire pour saisir les enjeux cachés derrière les façons de nommer. Cet article constitue une tentative d'incursion dans ce sujet complexe, ce labyrinthe de mots qui s'entrecroisent aux dépens de gens qui souffrent.

Cette brève réflexion nous porte à conclure que les professionnelles et les professionnels de l'aide ont beaucoup à perdre, en terme de contrôle social et de pouvoir de diagnostic, lorsqu'ils et elles remettent en question les vocables couramment utilisés dans le modèle médical ou psychosocial. On possède en donnant, écrivent Bourdieu et Passeron (1970. La main de celui qui donne est plus élevée que celle de celui qui reçoit, remarque Godbout (1992). Ainsi, plusieurs enjeux de pouvoir se cachent derrière les façons de désigner les gens avec qui nous travaillons. Cet exercice nécessite des mises en question, car on n'intervient pas de la même façon avec un patient qu'avec une victime, avec un cas qu'avec une personne qui s'identifie comme une détentrice d'expérience.

Il semble bien que le voir de l'observation (le voir sans être vu), celui qui définit des populations-cibles par exemple, ne soit parfois guère autre chose que le simple prolongement social $d u$ voir biologique initial, $d u$ «voir la proie», en somme un mode privilégié de l'agression sur l'autre (De Gaulejac et Roy 1993 : 118). 
Depuis les années 1980, plusieurs mouvements d'entraide et de solidarité ont démontré que l'aide peut prendre d'autres formes et d'autres noms. Les mouvements antipsychiatriques, les associations de défense de droits, les réseaux communautaires de conscientisation ont-ils encore envie de prendre part aux exercices de diagnostics et aux plans d'intervention, pour «aider» les professionnelles et professionnels à voir autrement, pour tenter de nommer autrement, pour aider autrement? Peut-être veulentils tous simplement ne plus participer à des exercices qui ne représentent ni ce qu'ils et elles sont, ni ce qu'ils et elles font, ni ce qu'ils et elles veulent devenir?

\section{Bibliographie}

ALARY, Jacques (dir. de publ.) (1988). Solidarités : pratique de recherche-action et de prise en charge par le milieu, Montréal, Éditions du Boréal Express.

APFELDORFER, Gérard (1993) (1991). Je mange, donc je suis: surpoids et troubles du comportement alimentaire, Paris, Éditions Payot et Rivages.

AUTÈS, Michel (1992). Travail social et pauvreté, Paris, Syros-alternatives.

AYRES, Beverley et Joseph LAGEY (1970). «Community Treatment Programs for Multi-Problem Families», dans Benjamin Schlesinger (sous la dir. de), The Multi-Problem Family : A Review and Annotated Bibliography, Toronto : University of Toronto Press, 55-71.

BECKER, Howard S. (1985) (1963). Outsiders : études de sociologie de la déviance, Paris, A.M. Métailié.

BELPAIRE, François (1994). Intervenir auprès des jeunes inadaptés sociaux : approche systémique, Montréal, Éditions du Merisier.

BIRT, Charles (1956). «The Family-centered project of Saint-Paul», Social Work, no d'octobre, 41-47.

BLAIS, Louise (1998). Pauvreté et santé mentale au féminin : l'étrangère à nos portes, Ottawa, Les Presses de l'Université d'Ottawa.

BOURDIEU, Pierre et Jean-Claude PASSERON (1970). La reproduction, Paris, Éditions de Minuit. BREGGIN, P. (1991). Toxic Psychiatry. New York, St-Martin's Press.

CAILLE, Philippe (1985). Familles et thérapeutes: lecture systémique d'une interaction, Paris, Les Éditions ESF.

CHESLER, Phyllis (1979). Les femmes et la folie, Paris, Payot.

CLÉMENT, René (1993). Parents en souffrance, Paris, Éditions Stock-Laurence Pernoud.

COOPER, D. (1970). Psychiatrie et anti-psychiatrie, Paris, Seuil.

CORIN, Hélène, Gilles BIBEAU,Jean-Claude MARTIN et Robert LAPLANTE (1990). Comprendre et soigner autrement : la régionalisation comme choix radical, Montréal, Unité de recherche psychosociale, Centre hospitalier Douglas.

DE GAULEJAC,Vincent et Isabel Taboada LÉONETTI (1994). La lutte des places, Paris, Desclés de Brouwer. 
DE GAULEJAC,Vincent et Shirley ROY (dir. de publ.) (1993). Les auteurs du lien, Paris, Éditions Hommes et perspective.

DIEDERICH, Nicole (1991). «Modèles d'inconduites et adaptation sociale paradoxale ou la déviance normalisée», Comportement humain, vol. 5, no 1,11-17.

EIGUER, Alberto (1987). La parenté fantasmatique, Paris, Dunod.

GEISMAR, Ludwig et Katherine WOOD (1960). Families at Risk :Working with Multiproblem Families, New York, Human Sciences Press.

GODBOUT, Jacques (1992). L'esprit du don, Montréal, Éditions Boréal.

GOFFMAN, Erving (1975). Stigmate : les usages sociaux des handicaps, Paris, Minuit.

FULLER, Mary Louise et Stephen GROCE (1991). «Obese Women's Responses to Appearance Norms», Free Inquiry in Creative Sociology, vol. 19, no 2, 167-174.

GHYSENS,A.C. (1986). «De l'assistance individuelle à l'action communautaire», Dis-moi qui tu aides, Namur, Presses universitaires de Namur, 29-39.

GOVE, Walter (1980). The Labelling of Deviance : Evaluating a Perspective, Sage Publications, Beverly Hills-London.

ILLICH, Ivan (1975). Némésis médicale, Paris, Seuil.

JONES, Lizanne et Raymond COCHRANE (1981). «Sterotypes of Mental Illness : A Test of the Labeling Hypothesis», The International Journal of Social Psychiatry, vol. 27, no 2, 92-104.

KIRK, Stewart et Herb KUTCHINS (1998). Aimez-vous le DSM? Le triomphe de la psychiatrie américaine. Le Plessis-Robinson, Institut Synthélabo.

LARCH, Christopher (1965). The Social Thought of Jane Addams, New York, Bobbs-Merrill Co. Inc.

LINK, Bruce G., Francis T CULLEN, Frank JAMES et John F. WOZNIAK (1987). «The Social Rejection of Former Mental Patients : Understanding Why Labels Matter», American Journal of Sociology, vol. 92, no 6, Mai, 1461-1502.

MEYER, Carol (1963). «Individualizing the Multiproblem Family», Social Casework, mai, 267-272.

MINUCHIN, S. (1974). Families and Family therapy, Cambridge, Harvard Press.

NOLLER, Patricia et Mary Anne FITZPATRICK (1993). Communication in Family Relationships, New Jersey, Prentice-Hall Inc.

OVERTON, Alice (1953). «Serving Families Who Don't Want Help», Social Casework, vol. XXXIV, 304-309.

PLATTEAU, Jean-Philippe (1986). «Fonction euphémisante et mystificatrice de l'aide», Dis-moi qui tu aides, Namur, 183-218.

RICHMOND, Mary (1965). Social Diagnosis, New York, The Free Press.

ROSEN,Alan (1994). «100\% Mabo :De-Colonising People with Mental Illness and Their Families», Australian and New Zealand Journal of Family Therapy, vol.15, no 3, Sept, 128-142.

ST-AMAND, Nérée (1985). Folie et oppression, Moncton, Les éditions d'Acadie.

ST-AMAND, Nérée et Michèle KÉRISIT (1998). Pauvreté et nouvelles solidarités : repenser l'intervention, Montréal, Éditions Saint-Martin, 1998.

SZASZ, Thomas (1976). Idéologie et folie, Paris, Presses universitaires de France.

TINKER, Katherine (1959). "Casework with Hard-to-Reach Families», American Journal of Orthopsychiatry, vol. 29, no 1,165-171. 
VANWORMER, Katherine (1989), «Co-dependency : Implications for Women and Therapy», Women and Therapy, vol. 8 , no 4.

WORRALL, Anne (1990). Offending Women, London, Routledge.

WRIGHT, Charles (1984). Constructions of Deviance in Sociological Theory: The Problem of Commensurability, Lanham, University Press of America. 